\title{
An Improved Contour Tracing Method of Extracting Shoreline Based on
}

\section{LiDAR Data}

\author{
Cai-xia $\mathrm{Yu}^{1, a}$, Yi-long $\mathrm{Li}^{2, b}$, Jun $\mathrm{Xu}^{1,3, \mathrm{c}}$, Wen-qian Huang ${ }^{1,4, \mathrm{~d}}$ \\ ${ }^{1}$ Dalian Navy Academy, Dalian 116018, China \\ ${ }^{2}$ Troops.75838, Guangzhou 510650, China \\ ${ }^{3}$ Chinese Academy of Surveying and Mapping, Beijing 100830, China \\ ${ }^{4}$ State Key Laboratory of Geo-information Engineering, Xi'an 710054, China \\ ayucaixiaxj@163.com, byilong@126.com, xujuny_1999@163.com, huangwq@163.com
}

\section{Key words: LiDAR Shoreline MHWS contour rasterization ArcGIS}

ABSTRACT. The Contour tracing method can automate extraction of tidal datum referenced shorelines from light detection and ranging ( LiDAR) data. With this method, shorelines are derived easily, but they are too broken, meandering and trembly. So an improved contour tracing method is put forward in this paper. Firstly, detection outlires is applied to the LiDAR data, and then the LiDAR data is rasterized by the reasonable resolution, lastly, the shorelines based on MHWS ( mean high water springs) datum surface, whose height calculated by the tide gauge data or tidal model are extracted from the raster data. An application shows that the improved method is more efficient than the contour tracing method, and the shorelines extracted are more smooth and reasonable.

\section{Introduction}

LiDAR can acquire high precision and density terrain surface three-dimension point clouds speedily, so it is of obvious advantage to surveying difficult area, such as coastal zone, and has become the new direction of coastal zone survey ${ }^{[1,2]}$. Currently, contour tracing methods which are simply and carried out easily, are adopted to extracting shorelines based on LiDAR point clouds in some foreign studies ${ }^{[3,4]}$. Shorelines created by contour tracing methods have more dithers, sawtooth-like and broken lines ${ }^{[1,4,5]}$, so some following procedures are further need to estimate, as well as smoothing which increased the workload. In the paper, an improved contour tracing method is developted to optimize the shoreline extracting process, and also improve shoreline quality.

\section{Contour Tracing Method}

According to national Surveying and Mapping Standard Criterion ${ }^{[6-8]}$ Shoreline is defined as mark line of the boundary of sea and land at the time of MHWS, which is the shoreline can be regarded as one contour intersected by MHWS and coast terrain. The contours have the same height value which is the height of MHWS, so shorelines can be transformed into the contour with MHWS height value. Then the main processes of extracting shorelines by contour tracing based on LiDAR point clouds are following, firstly, original LiDAR point clouds are filtered and classified to create coast digital elevation model (DEM), secondly, the regional MHWS height value is calculated by the tide gauge data or tidal model, lastly, the contours with MHWS height value are extracted by 
contour tracing method, namely the shoreline. Fig. 1 shows the flowchart of extracting shoreline with improved contour tracing method.

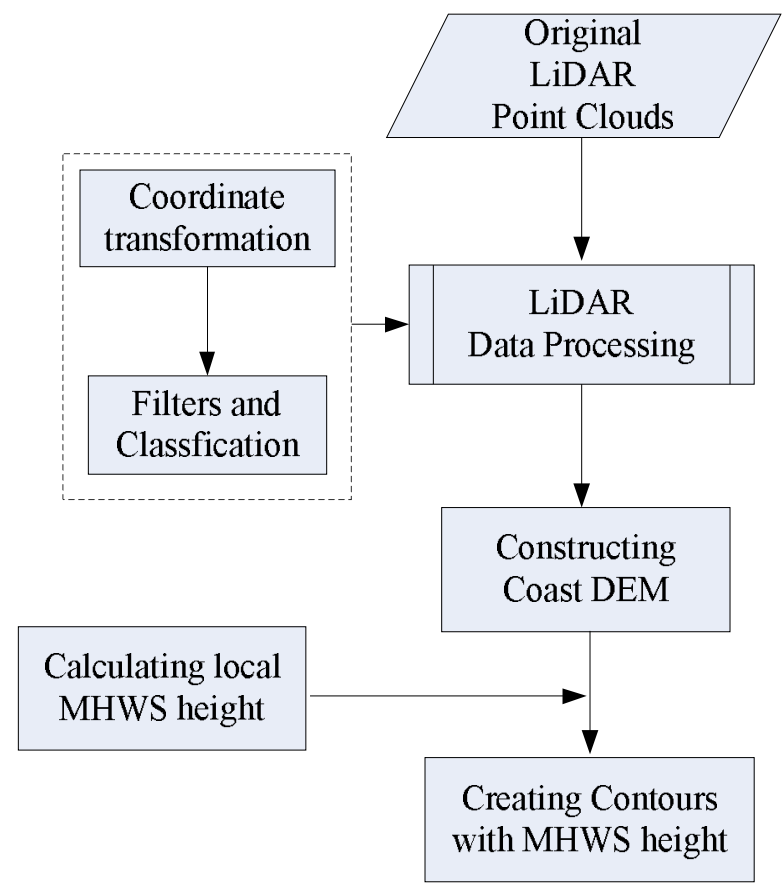

Fig.1. Flow chart of extracting shoreline with contour tracing method

By the above contour tracing method, the shoreline extracted is showed in fig. 2.

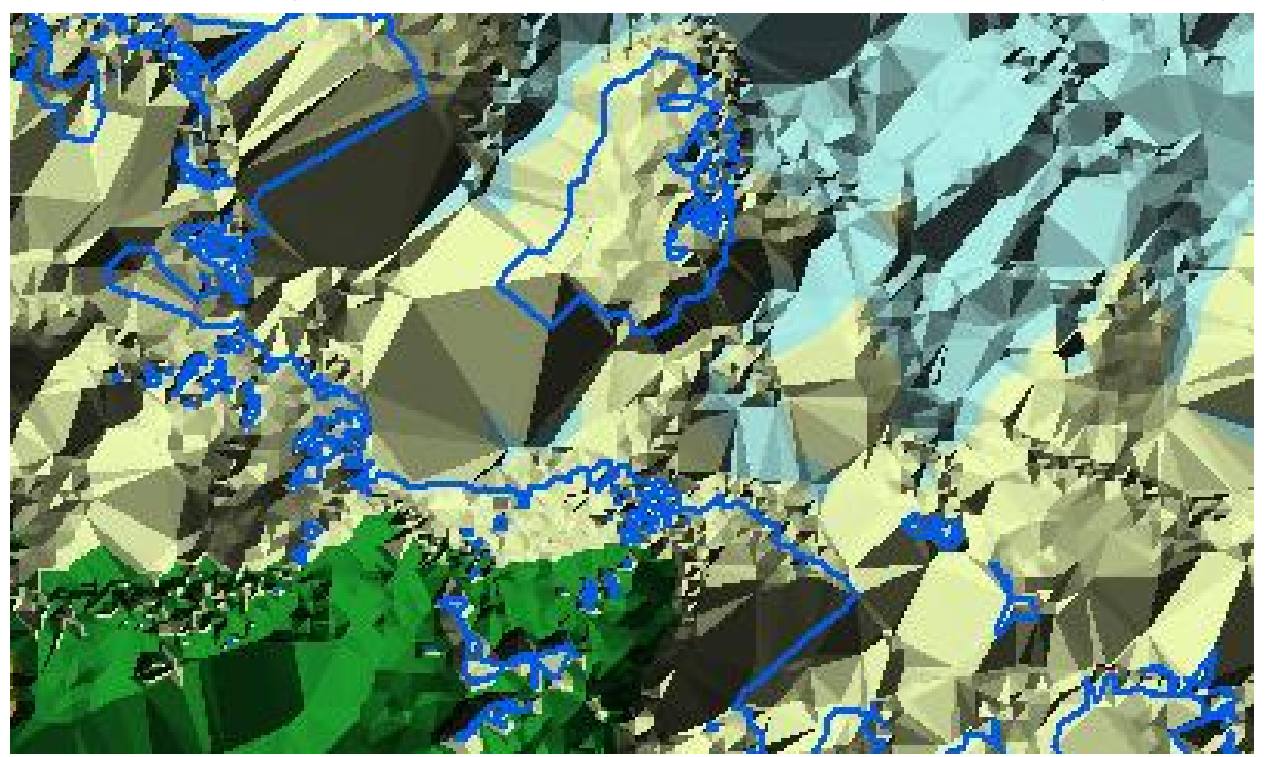

Fig.2. Shoreline extracted by contour tracing method loaded on coast DEM

It is evident that the shorelines are more meandering, trembley and broken; furthermore, there are many false lines or enclosed polygon, by Fig 2 . The main reason causing the circumstances such as Fig 2 are that the LiDAR data of seaside are influenced by wave and tide, the algae and seaweed on shallow are too thick, the noise especially outlies, and lack of data on strait or swale, and so on ${ }^{[1,}$ $5,9]$

So much more following manual works are needed to modify which bring higher labor intensity, lower efficiency, and also the shorelines extracted are easily influenced by subjective factors as well. In addition, the method is restricted by two aspects such as follows:

(1) Filtering and classification of LiDAR point clouds 
Currently, there are many studies on LiDAR point clouds filtering, but yet no method is universal and suitable to the complex terrain landform ${ }^{[10,11]}$, meanwhile, when filtering, it need continuous tests to adjust threshold, which consume the much longer time.

(2) Creation of coast DEM

LiDAR point clouds data are of random, high-density and high-precision, and coast terrain are complex as well, so the coast DEM creation is complex and time-consuming, meanwhile, it exits error and unreasonable.

\section{The improved Contour Tracing Method}

In order to improve shoreline quality, the paper improved the contour tracing method, first, detection outlires is applied to the LiDAR data; second, established LAS dataset based on ArcGIS ${ }^{[12 \text {, }}$ 13]; third, rasterization Terrain dataset or LiDAR point clouds to found much smoother coast terrain; at last, much reasonable shorelines extracted by raster surface. Fig. 3 shows the flowchart of extracting shoreline with improved contour tracing method.

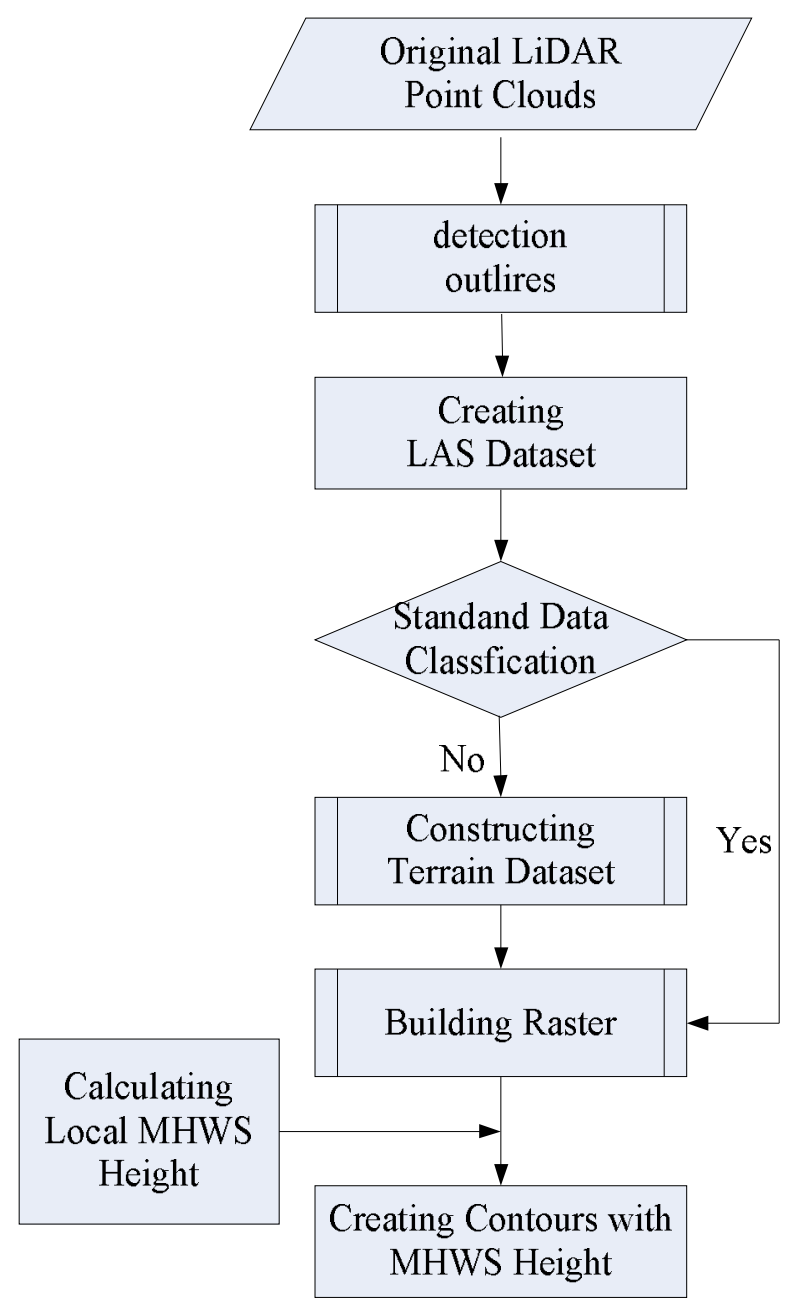

Fig.3. Flow chart of extracting shoreline with improved contour tracing method

(1) Detection outlires. Because of LiDAR characteristic, it is inevitable to bring noise into point clouds, including very high points, very low points and isolated point and so on ${ }^{[10,14]}$, all of which are false point clouds. So they must be removed, otherwise leading serious error.

(2) Creation LAS Dataset. In software ArcGIS 10.2, three-dimension LiDAR point clouds are quickly read and displayed by LAS Dataset. Of course, LiDAR data with WGS 84 coordination are 
transformed into those with national geodetic system 2000 (CGCS 2000) and national vertical datum1985.

(3) Judging whether LiDAR point clouds are classified or not. If true, it can go into procedure 5 directly, or else, go into procedure4.

(4) Construction Terrain dataset. In order to thinning data and reducing high frequency noise, it needs to choose the suitable Terrain pyramid type and the reasonable grade.

(5) Establishment raster surface data. Coast terrain surfaces are smoothed by interpolation method. If the previous procedure is 4 , raster surfaces are established by Terrain dataset, or else, by LAS Dataset directly.

(6) Calculation MHWS Height value. Local MHWS Height value of the coast area is calculated by the tide gauge data or tidal model ${ }^{[15,16]}$, and then transformed into national vertical datum1985.

(7) Extraction shoreline. In the coast terrain raster surface, height information are expressed in different grey level or color level, the contours with MHWS height value are built by raster surface, namely, shoreline.

\section{Experiment and Analysis}

\section{Experiment}

One certain coast in Dalian is chosen in the paper, ship-borne LiDAR point clouds data with $10 \mathrm{~cm}$ space are adopted, and there are about 220000 point clouds. Fig. 4 is the real-color point clouds, according to the above procedures; LAS Dataset is established in Fig. 5.

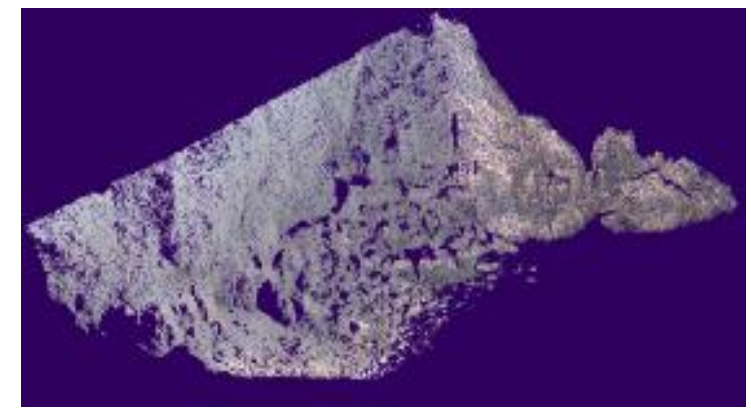

Fig.4 Real-color point clouds of the certain coast

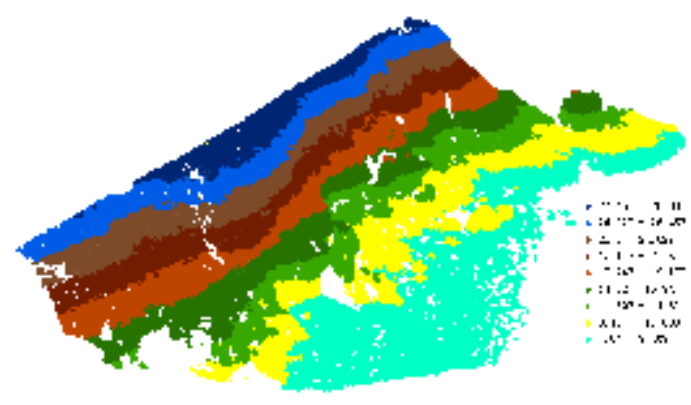

Fig.5 established LAS Dataset

Shorelines extracted by contour tracing method are loaded on raster data, like Fig. 6, meanwhile, Fig. 7 is the shorelines extracted by improved contour tracing method, which are loaded on raster data. Shorelines by these two different methods are overlaid showing in Fig. 8, blue lines expressing shorelines by contour tracing method, while red lines meaning ones by the improved methods. These three figures are all local magnified area of shorelines.

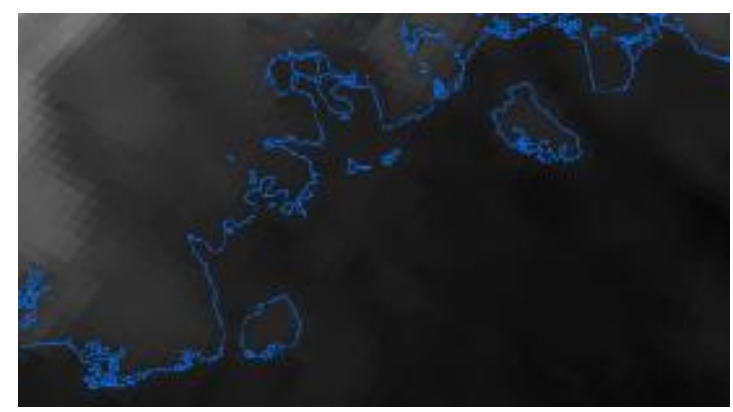

Fig. 6 Shorelines by contour tracing method

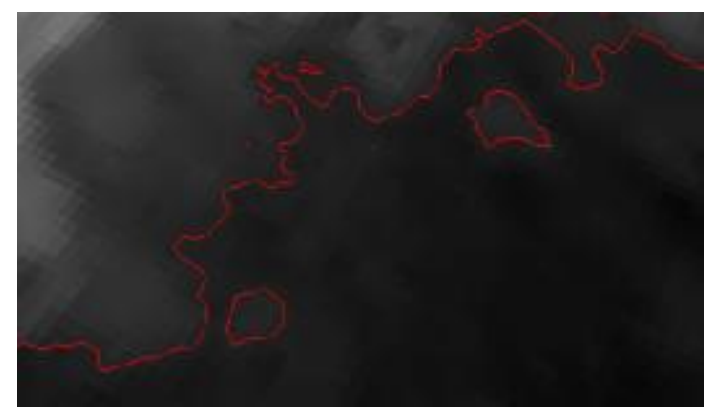

Fig. 7 Shorelines by improved method 


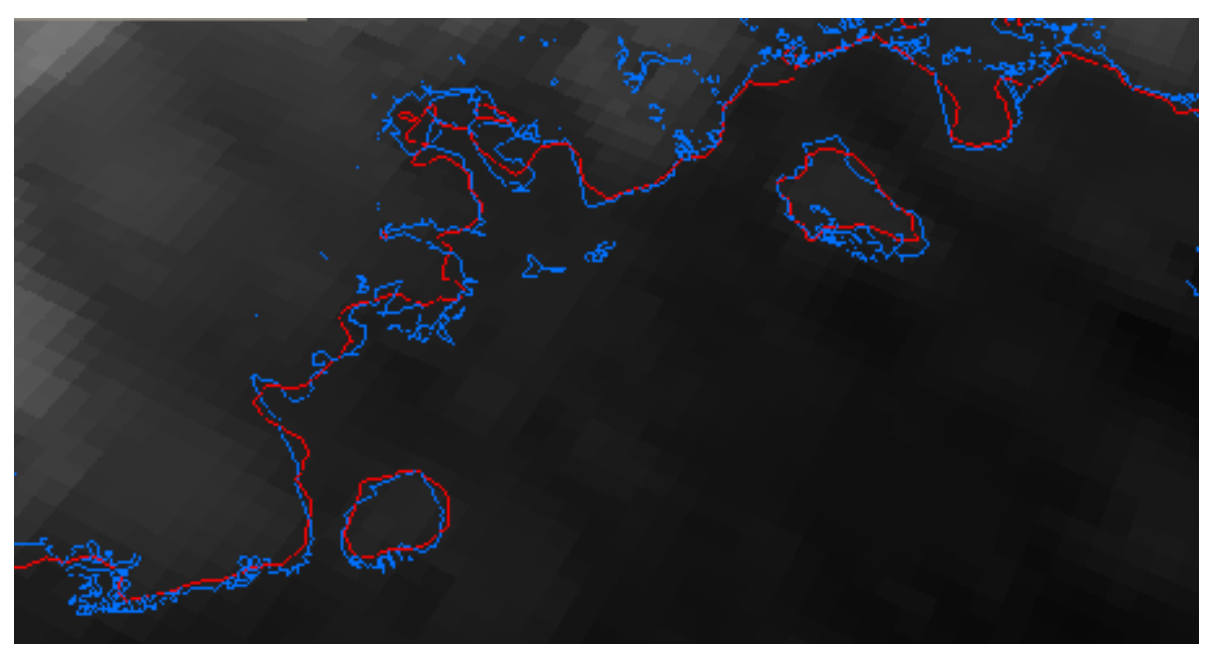

Fig. 8 The overlay of shorelines by two different methods

\section{Analysis}

In fact, it is very hard to create standard contours by the contour tracing method based on traditional data source which are sparse-density data, say nothing of high-precision, high- density and random collected LiDAR data, with the addition of the complex terrain of sea and land interface, the process of extracting shorelines are more complex and difficult, and yet shorelines are trembly, sawtooth-like, and there are many broken lines and isolated polygons (see Fig. 6 and Fig. 8). Moreover, by the improved method, error are eliminated firstly, then point clouds are rasterized which means the point clouds height are quantized again to be equilibrium, viz. filtering the high frequency noise, so shorelines showed in Fig. 7are much more smoother and suitable for the real coast area (see Fig. 8), of course, the improved method effectively reduces the following works and labor intensity, as well as improves the efficiency of shoreline surveying and mapping.

\section{Conclusions}

Confirming shorelines speedily and precisely is one important mission of Hydrography and Cartography at all times. The paper put forward an improved contour tracing method, which spurning coast DEM construction process in contour tracing method to guarantee much simpler and higher efficiency. Especially, LiDAR point clouds are rasterized, so shorelines extracted from raster data are much smoother and reasonable. It tested that the improved methods can be applied to create shorelines in digital chart production.

\section{Acknowledgement}

National Natural Science Foundation Youth Fund (41501500) ; Opening Fund of State Key Laboratory of Geo-information Engineering (SKLGIE2016-M-4-1)

\section{Reference}

[1] Yu Caixia, Wang jiayao, Xujun, et al. Advance of Coastline Extraction Technology [J].Jounal of Geomatics Science and Technology, 2014, 31(3):305-309.

[2] Stockdon H, Sallenger A, Holman R et al. Estimation of Shoreline Position and Change Using Airborne Topographic Lidar Data[J]. Journal of Coastal Research, 2002, 18(3): 502-513.

[3] Robertson W V, Whitman D, Zhang K Q et al. Mapping Shoreline Position Using Airborne Laser Altimetry [J]. Journal of Coastal Research, 2004, 20(3): 884-892. 
[4] Leatherman, S.P. Shoreline change mapping and management along the U.S. east coast [J]: Journal of Coastal Research Special, 2003(38): 5-13.

[5] Yu Caixia, Huang Wenqian, Zheng Yidong, et al. A Method for Creating Contours from LiDAR Based on ArcGIS [J]. Hydrographic Surveying and Charting, 2015, 35(2): 52-54.

[6] State Bureau of Quality and Technical Supervision. Hydrographic Survey Criterion [S]. Beijing: China Standard Press, 1999.

[7] State Bureau of Quality and Technical Supervision. Symbols, Abbreviations and Terms used on Chinese Charts [S]. Beijing: China Standard Press, 1999.

[8] State Bureau of Quality and Technical Supervision. Topographic Map Symbols [S]. Beijing: China Standard Press, 1999.

[9] Hongxing Liu, Douglas Sherman, Songgang Gu. Automated Extraction of Shorelines from Airborne Light Detection and Ranging Data and Accuracy Assessment Based on Monte Carlo Simulation [J]. Journal of Coastal Research, 2007, 23(6): 1359-1369.

[10]Zhang Xiaohong. Theory and Method of Airborne Laser Detection and Ranging [M].Wuhan: Wuhan University Press, 2007.

[11]Axelsson P. DEM Generation form Laser Scanner Data Using Adaptive TIN Models [J]. IAPRS, 2000, 33(B4):110-117.

[12]LiDAR in ArcGIS 10.1 for Desktop. Esri China Information Technology Co. Ltd. 2012.

[13] ArcGIS Help 10.2, USA: ESRI, 2013.

[14]Lai Xudong. Theory and Application of Airborne Laser Detection and Ranging [M]. Beijing: Publishing House of Electronics Industry, 2010.

[15]Bao Jingyang, $\mathrm{Xu}$ Jun. Tide Analysis from Altimeter data and the Establishment and Application of Tide Model [M]. Beijing: Surveying and Mapping Press, 2013.

[16]Dang Yamin, Cheng Pengfei, Zhang Chuanying, et al. Technology and Method of Island Surveying and Mapping [M]. Beijing: Surveying and Mapping Press, 2012. 